\title{
Capilaroscopia Periungueal: Importância para a Investigação do Fenômeno de Raynaud e Doenças do Espectro da Esclerose Sistêmica(*)
}

\section{Importance of the Nailfold Capillaroscopy in the Investigation of Raynaud's Phenomenon and Scleroderma-Spectrum Syndromes}

\author{
Cristiane Kayser( ${ }^{(1)}$ e Luís Eduardo Coelho Andrade( ${ }^{(2)}$
}

\begin{abstract}
RESUMO
A capilaroscopia periungueal é um exame não invasivo e extremamente útil para avaliação do componente morfológico da microcirculação. Permite o diagnóstico diferencial precoce entre indivíduos com fenômeno de Raynaud (FRy) primário e secundário. $\mathrm{Na}$ esclerose sistêmica e síndromes correlatas ocorrem alterações capilaroscópicas facilmente distinguíveis do padrão encontrado em indivíduos normais, sendo caracterizadas pela presença de ectasia capilar e áreas de deleção vascular, além de diminuição difusa da quantidade de alças. Em razão de sua relevância diagnóstica, a familiarização do médico clínico e do reumatologista com a CPU é de fundamental importância.
\end{abstract}

Palavras-chave: capilaroscopia periungueal, microcirculação, esclerose sistêmica, fenômeno de Raynaud (FRy).

\section{INTRODUÇÃO}

A capilaroscopia periungueal (CPU) é um método simples, não invasivo e de baixo custo, que permite o acesso in vivo da microcirculação através da visualização direta da fileira distal de capilares da região periungueal dos dedos das mãos. É um método extensamente aceito para avaliação da microvasculatura e investigação diagnóstica das doenças reumáticas auto-imunes e síndromes que cursem com fenômeno de Raynaud (FRy).

A primeira descrição de visualização capilar com um sistema de magnificação óptica parece ser de J. C. Kolhaus,

\begin{abstract}
Nailfold capillaroscopy is a noninvasive method useful for the evaluation of microcirculation morphologic component. It allows an early differential diagnosis between subjects with primary Raynaud's phenomenon and patients with secondary Raynaud's phenomenon. In systemic sclerosis and sclerodermaspectrum syndromes there are capillaroscopic findings easily distinguished from the pattern of healthy controls, and characterized by the presence of enlarged loops, avascular areas and diffuse loss of capillaries. Because of their diagnostic relevance, the acquaintance of internists and rheumatologists with nailfold capillaroscopy is of utmost importance.
\end{abstract}

Keywords: nailfold capillaroscopy, microcirculation, systemic sclerosis, Raynaud's phenomenon.

em 1663. No início do século 20, vários investigadores descreveram o uso da CPU em diversas doenças, entre elas a esclerose sistêmica ${ }^{(1)}$. A maioria desses estudos, entretanto, empregava microscópios com grande aumento (100 a 300 vezes), com resultados pouco reprodutivos e inconsistentes. Essa abordagem resultou em uma pletora de publicações sobre achados capilaroscópicos espúrios em uma grande diversidade de enfermidades, o que fez com que a CPU passasse a ser negligenciada nas décadas seguintes. Na década de 50, Wertheimer e Wertheimer ressaltaram a importância de uma abordagem panorâmica, com aumentos moderados de cerca de 20 vezes, que possibilitariam observações mais

\footnotetext{
Disciplina de Reumatologia, Escola Paulista de Medicina, Universidade Federal de São Paulo (EPM-UNIFESP). Trabalho recebido em $11 / 08 / 03$. Aprovado, após revisão, em 05/01/04.

1. Pós-graduanda em nível de doutorado em Medicina. EPM-UNIFESP.

2. Professor Adjunto - Livre Docente. EPM-UNIFESP.
}

Endereço para correspondência: Dra. Cristiane Kayser. Rua Botucatu 740, 3ªndar, CEP 04023-062, São Paulo, SP. E-mail: criskayser@hotmail.com 
consistentes e reprodutíveis. Porém, foi na década de 70 que Maricq et al. realizaram extensos estudos com capilaroscopia feita sob moderada magnificação (10 a 50 vezes), permitindo a visão panorâmica dos capilares periungueais e estabelecendo as vantagens da CPU na investigação do FRy e síndromes reumáticas auto-imunes. Os autores descreveram alterações morfológicas características associadas às doenças do tecido conjuntivo e estabeleceram em sucessivas publicações o padrão capilaroscópico associado à esclerose sistêmica $(\mathrm{ES})^{(2-4)}$. Em seguida, vários autores em todo o mundo confirmaram os achados inicialsmente descritos, com progressiva popularização do método. Na década de 80, a CPU foi introduzida no Brasil mediante um estudo em 800 indivíduos hígidos e padronização do método para a nossa população ${ }^{(5,6)}$.

Apesar disso, observa-se pouca intimidade com o exame entre os clínicos e até mesmo entre os reumatologistas. O objetivo deste artigo é descrever as alterações capilaroscópicas mais freqüentemente encontradas em pacientes com doenças reumáticas, dando-se especial destaque à ES, auxiliando o clínico na interpretação do exame. Faremos ainda, uma breve revisão dos aspectos mais atuais da literatura em relação à CPU. Daremos também destaque a imagens de CPU para familiarizar o leitor com as mesmas.

\section{MÉTODOS DE REALIZAÇÃO DO EXAME}

A CPU pode ser realizada com vários equipamentos incluindo-se o oftalmoscópio, a lupa estereomicroscópica e equipamento computadorizado para videocapilaroscopia em tempo real. Além do instrumento óptico, deve-se utilizar uma epiiluminação que incida a $45^{\circ}$ sobre a superfície da pele. A videocapilaroscopia geralmente associa à $\mathrm{CPU}$ convencional um sistema de vídeo acoplado a um computador com um programa para analisar alguns parâmetros em alças individuais ${ }^{(7,8)}$. A CPU convencional com lupa estereomicroscópica e avaliação panorâmica dos capilares sob magnificação de 10 a 25 vezes parece ser o método com melhor reprodutibilidade e com menor custo, sendo o método de escolha em nosso meio.

$\mathrm{Na} \mathrm{CPU}$, após a aplicação de um meio oleoso sobre a superfície da pele, são visualizados o plexo venoso subpapilar (PVS) e os capilares das papilas dérmicas. A realização deste exame na região periungueal dá-se pelo fato de que na fileira capilar distal desta região, as papilas dérmicas estão dispostas de forma paralela à superfície cutânea, permitindo a visualização dos diversos segmentos capilares (aferente, de transição e eferente) de forma horizontal, enquanto na maior parte do tegumento as papilas são perpendiculares à superfície da pele ${ }^{(5)}$. Todos os dedos das mãos devem ser avaliados. Para obtenção de resultados mais acurados, o paciente deve estar confortável e tranqüilo, especialmente do ponto de vista térmico. Fumantes devem se abster do cigarro nas 6 horas que antecedem o exame. Esses cuidados visam prevenir possível vasoconstrição que venha interferir com a plena visibilidade dos capilares periungueais.

\section{ACHADOS CAPILAROSCÓPICOS EM INDIVÍDUOS NORMAIS}

Em indivíduos normais as alças capilares são de tamanho, forma e cor homogêneas, dispostas transversalmente ao longo da cutícula (Figura 1). Os capilares normais podem apresentar variações morfológicas discretas, tais como alças tortuosas ou enoveladas (com entrecruzamentos) (Figura 2). O plexo venoso subpapilar pode ser visualizado em extensão variável em aproximadamente $60 \%$ da população geral, apresentando maior visibilidade em crianças e em pessoas de pela clara (Figura 3). Curiosamente, o plexo venoso apresenta maior visibilidade nos dedos da mão esquerda na maior parte das pessoas. O número de capilares varia de $7 / \mathrm{mm}$ a $12 / \mathrm{mm}$. Em alguns casos, podem-se encontrar capilares ectasiados (dilatados), mas não é comum o encontro de desvascularização (deleção). Achados isolados de deleção em indivíduos normais podem representar microcicatrizes na região periungueal. Micropetéquias de distribuição focal também podem estar presentes e parecem estar associadas com microtraumas cotidianos ${ }^{(6)}$.

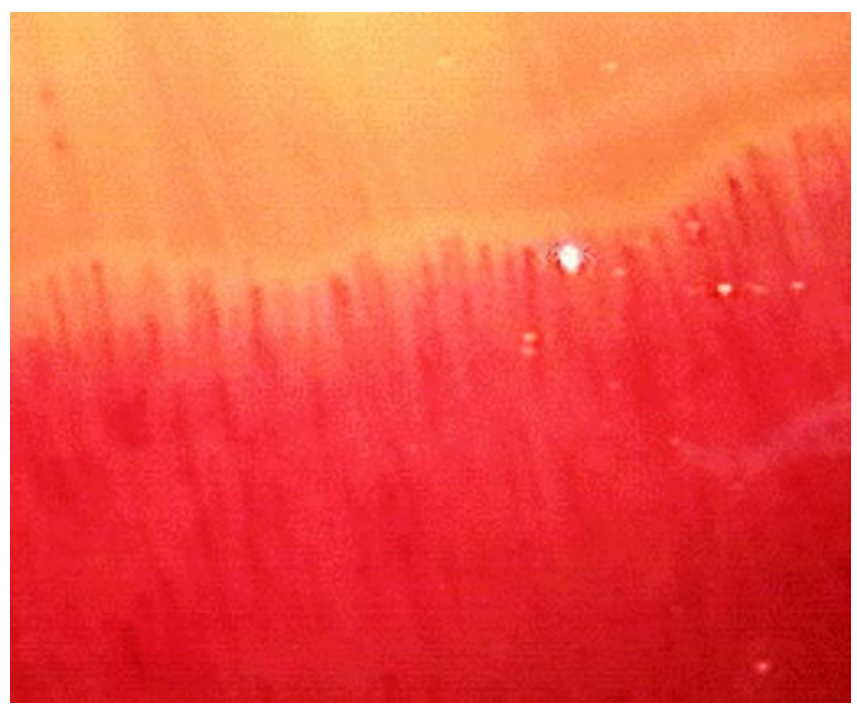

FiguRA 1 - Padrão capilaroscópico normal. Capilares com forma, tamanho e distribuição homogêneas. 
É válido lembrar que alguma variabilidade é encontrada entre indivíduos normais e mesmo entre os dedos de um mesmo indivíduo. O examinador deve estar habituado com esta variabilidade para não diagnosticar equivocadamente anormalidades discretas e isoladas como microangiopatia ${ }^{(8)}$.

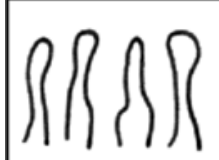

ABERTO

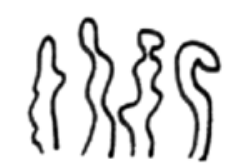

TORTUOSO

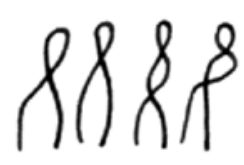

CRUZADO

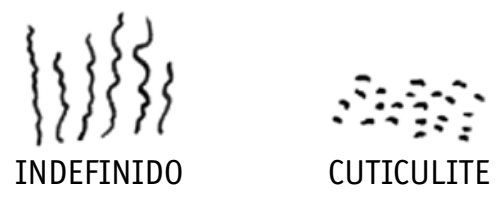

Figura 2 - Padrões morfológicos dominantes.

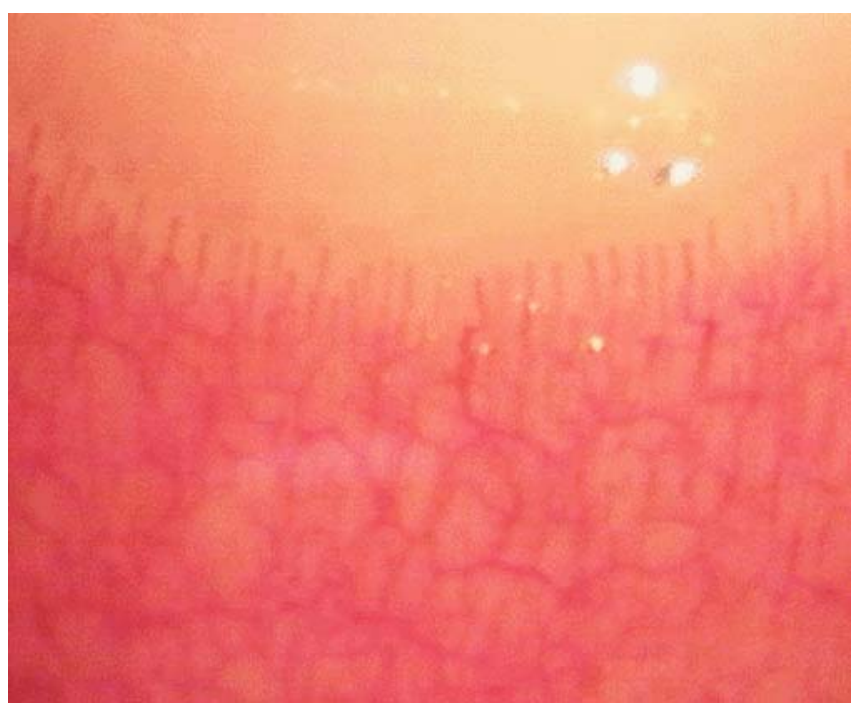

FiguRa 3 - Capilaroscopia periungueal em indivíduo normal com plexo venoso subpapilar visível (trama de vasos na região proximal à fileira capilar distal).

PARÂMETROS PARA AVALIAÇÃO DA MICROANGIOPATIA Alterações na forma e arranjo das alças capilares são as informações mais importantes para o diagnóstico e acompanhamento das microangiopatias. Vários métodos e formas de avaliação são descritos na literatura para interpretação das alterações vistas à CPU. Em nosso serviço, utilizamos uma combinação dos métodos de avaliação panorâmica da CPU com a forma analítica de interpretação ${ }^{(6,9)}$. Os principais parâmetros são analisados de forma quantitativa ou semiquantitativa quanto à presença de anomalias microvasculares. Os principais elementos analisados em cada dedo estão listados na Tabela 1.

TABELA 1

ParÂMEtros de AVAliaÇão DA MiCroAngiopatia

Número de petéquias e padrão de distribuição (focal ou difuso)

Número de capilares por mm

Grau de deleção

Número de capilares ectasiados

Número de megacapilares

Em razão de sua relevância para a definição das microangiopatias associadas às doenças reumáticas e principalmente à ES, deve-se dar especial atenção à presença de dilatação capilar e desvascularização. O capilar é considerado ectasiado quando as alças encontram-se alargadas em todos os seus 3 ramos - aferente, transição e eferente, com tamanho de 4-9 vezes maior que o normal ${ }^{(1,9)}$. Megacapilares correspondem a alças extremamente alargadas, com calibre 10 ou mais vezes maior que as alças adjacentes normais ${ }^{(1)}$. Para o registro de capilares ectasiados e megacapilares conta-se o número de capilares com tais alterações em cada dedo.

A desvascularização capilar pode ser focal ou difusa. A desvascularização difusa é avaliada através da contagem do número de capilares $/ \mathrm{mm}$. Diz-se que há desvascularização difusa quando a contagem do número de capilares for menor que $7 / \mathrm{mm}$. Para quantificar o grau de deleção focal, ou área avascular, seguimos a escala de Lee et al.(1983) ${ }^{(10)}$ que definem como deleção a ausência de dois ou mais capilares sucessivos, permitindo quantificar este parâmetro em uma escala de $0 \mathrm{a}$ 3 conforme a extensão das lesões:

0 - nenhuma área de deleção;

1 - uma ou duas áreas de deleção descontínuas;

2 - mais de duas áreas de deleção descontínuas;

3 - áreas extensas e confluentes de desvascularização.

\section{O PADRÃO SD E OUTROS ACHADOS NAS DOENÇAS REUMÁTICAS}

Alterações morfológicas na microvasculatura periungueal são bem descritas na ES e muitas vezes surgem nas fases iniciais da doença, quando o FRy é ainda a única mani- 
festação clínica ${ }^{(4,11,12)}$. O característico conjunto de alterações capilaroscópicas presentes na ES foi denominado padrão $\mathrm{SD}$, por Maricq et al., e está presente em aproximadamente 95\% dos casos de ES, mas pode ser observado também na dermatomiosite e na doença mista do tecido conjuntivo (DMTC), nesta última principalmente se existe expressão de manifestações esclerodérmicas ${ }^{(3,10,13)}$.

$\mathrm{Na}$ ES, além da presença de capilares ectasiados e perda de capilares ou áreas de desvascularização, ocorre uma perda dramática da aparência normal do leito capilar, com uma desestruturação da sua arquitetura capilar (Figura 4) $)^{(3,9)}$. Observa-se também freqüentemente um "aspecto borrado", com indefinição do contorno dos capilares, que se deve provavelmente ao edema intersticial e à proliferação anômala de tecido conjuntivo observados nessa doença. Esta característica ocasiona a impressão de que as imagens estão fora de foco. Micropetéquias com distribuição difusa podem estar presentes, estando relacionadas com dano endotelial (Figura 5).

Na DMTC e na dermatomiosite o padrão SD é encontrado em cerca de $60 \%$ dos $\operatorname{casos}^{(3,13,14)}$. Embora geralmente indistinguível das alterações encontradas na ES, a microangiopatia da dermatomiosite apresenta com maior freqüência capilares em forma de arbusto, com ramificações exuberantes (Figura 6). As alterações vistas na dermatomiosite também costumam ter um caráter mais dinâmico que na ES, podendo regredir rapidamente com o controle da doença, o que não costuma acontecer na ES, exceto após longo tempo de evolução. Curiosamente, não se costuma observar alterações à CPU na polimiosite.

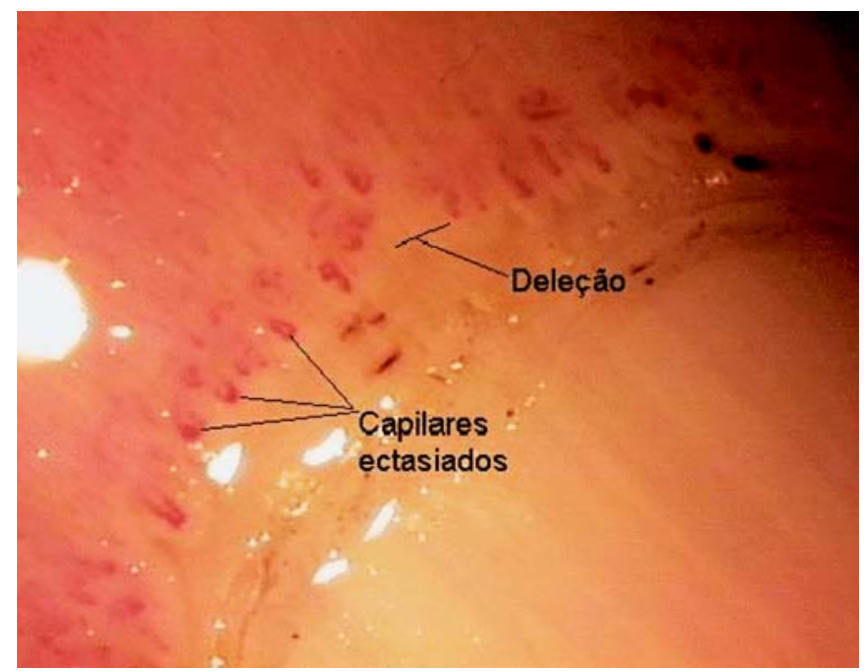

FIGURA 4 - Capilaroscopia periungueal com padrão SD em indivíduo com esclerose sistêmica.

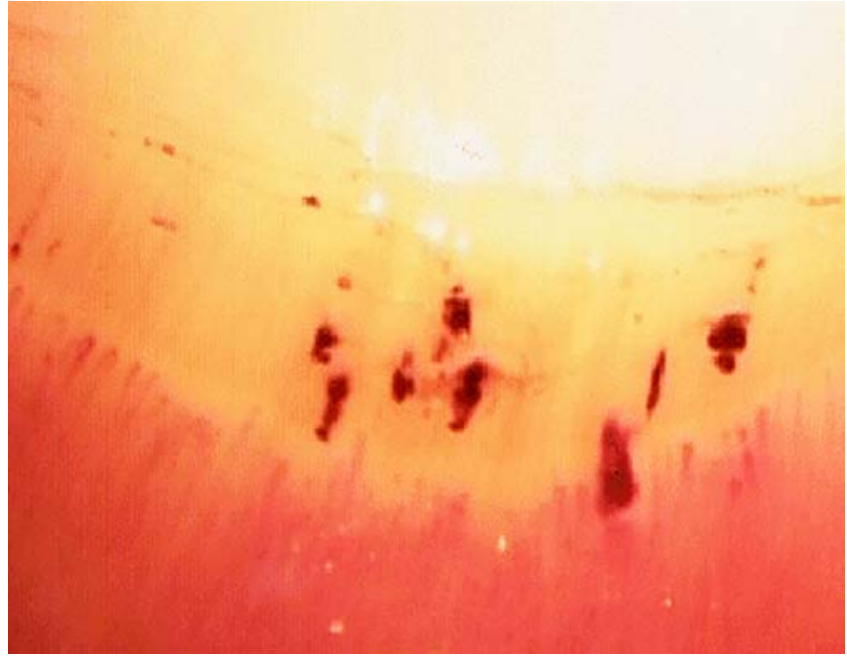

FiguRA 5 - Presença de micropetéquias na região periungueal.

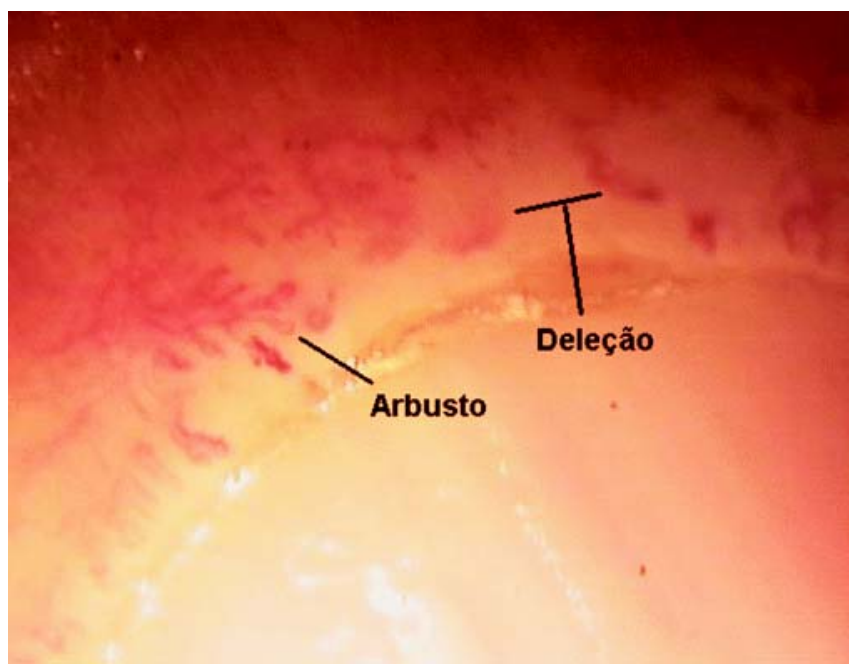

Figura 6 - Presença de capilares em arbusto, intensa desorganização da arquitetura capilar e áreas extensas de desvascularização em paciente com dermatomiosite.

No FRy primário, a regra é um padrão capilaroscópico semelhante ao normal ${ }^{(15)}$. Assim como nos indivíduos normais, podem ser observados alguns capilares dilatados, mas há ausência de megacapilares e desvascularização.

No lúpus eritematoso sistêmico (LES) podem ser observadas alterações capilaroscópicas exuberantes, porém menos específicas. Alterações tais como presença de alças alongadas, tortuosas e enoveladas são descritas em cerca de $30 \%$ dos 
pacientes, mas não são específicas dessa doença ${ }^{(13,16)}$. O padrão SD é raro, e, em aproximadamente 30\% dos pacientes, não se observa nenhuma alteração à CPU. A presença de dilatação capilar é mais encontrada em pacientes com LES que apresentam $\mathrm{FRy}^{(16)}$. A presença de lesão discóide comprometendo a região periungueal pode ocasionar um panorama capilaroscópico semelhante ao padrão SD convencional, mas tal eventualidade é facilmente identificada pelo exame macroscópico dos dedos. O padrão SD genuíno pode ser encontrado em 5\% a $10 \%$ dos pacientes lúpicos, estando associado à presença de anticorpos anti-U1-RNP, FRy e disfagia ${ }^{(17)}$. É possível que esta fração de pacientes represente um grau discreto de superposição entre LES e ES. Uma alteração característica do LES é a lesão em janela, usualmente visível na superfície extensora das falanges. Esta lesão caracteriza-se por desvascularização circunscrita de capilares das papilas dérmicas e extensa visibilidade do plexo venoso subpapilar subjacente.

$\mathrm{Na}$ artrite reumatóide não há descrição de padrão SD e em geral a rede capilar periungueal apresenta configuração normal ${ }^{(3,6)}$. Pode-se encontrar apenas alterações não específicas como tortuosidade capilar, aumento da visibilidade do plexo venoso subpapilar e micropetéquias.

\section{Aspectos atuais}

Nas últimas décadas vários autores têm dado destaque para a importância da capilaroscopia periungueal no diagnóstico precoce da esclerose sistêmica. A CPU tem sido considerada o método de escolha para a detecção precoce da microangiopatia típica da ES e síndromes correlatas e para o diagnóstico diferencial de indivíduos com FRy primário.

Em um estudo de meta-análise, Spencer-Green (1998) ${ }^{(18)}$ demonstrou que o fator com melhor valor preditivo positivo para evolução para uma desordem secundária em indivíduos com FRy primário foi a presença de alterações à CPU.

Segundo Maricq et al. (1982), o padrão SD, quando encontrado em pacientes com FRy isolado, confere um risco relativo de 12,9 para desenvolvimento de esclerose sistêmica $^{(4)}$. Luggen et al. (1995), ao acompanharem prospectivamente 64 pacientes com "suspeita" de FRy secundário, verificaram que a presença de anormalidades à CPU na primeira avaliação foi o fator com maior poder preditivo para o desenvolvimento de ES (risco relativo = 21,8); também foi o único fator independentemente associado com o desenvolvimento de qualquer doença reumática auto-imune (risco relativo $=8,3)^{(19)}$. Priollet et al. (1987) também acompanharam 73 pacientes com FRy primário (sem anormalidades clínicas, laboratoriais ou sorológicas associadas) ou com "suspeita" de FRy secundário por 4,7 \pm 1 ano $^{(20)}$. Neste último grupo se encontravam pacientes que não preenchiam critérios para diagnóstico de nenhuma doença reumática auto-imune segundo os critérios do Colégio Americano de Reumatologia (ACR), mas com pelo menos um achado sugestivo de doença reumática, como presença de anticorpos antinúcleo, úlceras digitais ou alterações à CPU. Dos 24 pacientes com FRy secundário "suspeito", 14 preencheram diagnóstico para alguma doença reumática autoimune ao final do acompanhamento, sendo que os fatores que se relacionaram com tal evolução foram: alterações à $\mathrm{CPU}$, presença de anticorpos antinúcleo, úlceras digitais e presença de "mãos em pão".

O fortalecimento da CPU para a avaliação do FRy é visível na literatura mais recente. Nas últimas décadas, diversos especialistas têm chamado a atenção para a existência de uma considerável fração de pacientes que clinicamente recebem o diagnóstico de ES, mas que não preenchem os critérios diagnósticos do ACR (1980) para o diagnóstico de $\mathrm{ES}^{(21)}$. Isto é particularmente aplicável aos pacientes com a forma limitada da doença, aos casos de ES sine scleroderma, e mesmo na formas difusa em seus estágios iniciais. Em 2001, LeRoy e Medsger Jr, na tentativa de identificar este subgrupo de indivíduos com algumas características de ES mas que não preenchem os critérios do ACR para o diagnóstico de ES, propuseram critérios para classificação da Esclerose Sistêmica Precoce ${ }^{(22)}$. Nesta classificação, o FRy deve estar obrigatoriamente presente, ainda que apenas por informação subjetiva, e deve estar associado à presença de microangiopatia SD à capilaroscopia periungueal ou à presença de auto-anticorpos considerados específicos para a ES (anticentrômero, anti-Scl-70, antifibrilarina, anti-PM-Scl, anti-RNA polimerase I ou III).

De fato, estudos mostram que a sensibilidade diagnóstica dos critérios do ACR para ES melhora sensivelmente com o acréscimo da CPU. Lonzetti et al. (2001), ao avaliarem 259 pacientes seguidos com diagnóstico clínico de ES, mostraram que, principalmente no subgrupo de pacientes com a forma limitada da doença, a sensibilidade diagnóstica dos critérios do ACR é baixa ${ }^{(23)}$. Nos pacientes com ES limitada, a sensibilidade diagnóstica aumentou de 33,6\% para $82,9 \%$ ao se acrescentar a presença de alterações à CPU (capilares dilatados e áreas de desvascularização) aos critérios diagnósticos do ACR.

Apesar de controverso, parece haver também uma correlação dos achados capilaroscópicos com o grau de acometimento visceral na ES e com o tempo de duração da doença ${ }^{(11,24-28)}$. Em um estudo realizado em nosso serviço 
com 29 pacientes com ES o acometimento esofágico (avaliado por esofagograma) e pulmonar (avaliado por raios X de tórax e prova de função pulmonar) se correlacionou com maior grau de desvascularização à $\mathrm{CPU}^{(25)}$. Mais recentemente, um estudo realizado por Bredemeier (2002) com 91 pacientes com ES encontrou uma correlação positiva com alterações à CPU e infiltrado em vidro fosco na tomografia de tórax de alta resolução ${ }^{(26)}$. Riccieri et al. (2003) encontraram uma correlação positiva entre os níveis séricos de IL-13 (uma citocina implicada na patogênese de várias doenças caracterizadas por fibrose) e anormalidades na CPU em pacientes com $\mathrm{ES}^{(29)}$. Por outro lado, alguns autores não encontraram tais correlações ${ }^{(10,11)}$. Tal variabilidade pode ser decorrente das diferenças metodológicas nos diversos estudos, como forma de aferição da microan-

\section{REFERÊNCIAS}

1. Silva LSM, Lima ARAG, Pucinelli MLC, Atra E, Andrade LEC: Capilaroscopia panorâmica periungueal e sua aplicação em doenças reumáticas. Rev Ass Med Brasil 43:69-73,1997.

2. Maricq HR, LeRoy EC: Patterns of finger capillary abnormalities in connective tissue disease by "wide-field" microscopy. Arthritis Rheum 16:619-28,1973.

3. Maricq HR, LeRoy EC, D,Angelo WA, et al: Diagnostic potential of in vivo capillary microscopy in scleroderma and related disorders. Arthritis Rheum 23:183-189,1980.

4. Maricq HR, Harper FE, Khan MM: Microvascular abnormalities as possible predictors of disease subsets in Raynaud's phenomenon and early connective tissue disease. Clin Exp Rheumatol 1: 195-205, 1982.

5. Andrade LEC, Atra E, Pucinelli ML, Ikedo F: Capilaroscopia periungueal: proposição de uma nova metodologia e aplicação em indivíduos hígidos e portadores de enfermidades reumáticas. Rev Bras Reumatol 30:71-81,1990.

6. Andrade LEC, Gabriel JrA, Assad RL, Ferrari JAL, Atra E: Panoramic nailfold cappilaroscopy: a new reading method and normal range. Semin Arthritis Rheum 20:21-31,1990.

7. Bukhari M, Hollis S, Moore T, Jayson MI, Herrick AL: Quantitation of microcirculatory abnormalities in patients with primary Raynaud's phenomenon and systemic sclerosis by video capillaroscopy. Rheumatology (Oxford) 39:506-12,2000.

8. Grassi W, Medico PD, Izzo F, Cervini C: Microvascular involvement in systemic sclerosis: capillaroscopic findings. Semin Arthritis Rheum 30:397-402,2001.

9. Maricq HR: Wide-field capillary microscopy. Technique and rating scale for abnormalities seen in scleroderma and related disorders. Arthritis Rheum 24:1159-65,1981.

10. Lee P, Leung F, Alderdice C, Armstrong SK: Nailfold capillary microscopy in the connective tissue diseases: a semiquantitative assessment. J Rheumatol 10:930-8,1983. giopatia, tamanho amostral e a própria heterogeneidade clínica observada nos pacientes com ES.

Pode-se concluir que a capilaroscopia periungueal é um instrumento valioso para investigação do FRy na prática clínica, permitindo o diagnóstico diferencial entre FRy primário e secundário. É também considerada útil para a avaliação da gravidade da doença e prognóstico em pacientes com ES.

Em razão de sua relevância diagnóstica, é extremamente desejável que médicos clínicos e reumatologistas estejam familiarizados com a CPU para poderem indicar a realização do exame quando necessário e para interpretar e apreciar os resultados advindos do mesmo. $\mathrm{O}$ treinamento de indivíduos capazes de executar a CPU nos diversos centros é fundamental neste processo.
11. Statham BN, Rowell NR: Quantification of the nailfold capillary abnormalities in systemic sclerosis and Raynaud's syndrome. Acta Derm Venereol (Stockl) 66: 139-43, 1986.

12. Ohtsuka T: Quantitative analysis of nailfold capillary abnormalities in patients with connective tissue diseases. Int J Dermatol 38: 757-64,1999.

13. Ohtsuka $\mathrm{T}$, Ishikawa $\mathrm{H}$ : Statistical definition of nailfold capillary pattern in patients with systemic sclerosis. Dermatology 188 : 286-9,1994.

14. Spencer-Green G, Crowe WE, Levinson JE: Nailfold capillary abnormalities and clinical outcome in childhood dermatomyositis. Arthritis Rheum 25:954-8,1982.

15. Houtman PM, Kallenberg CG, Fidler V, Wouda AA: Diagnostic significance of nailfold capillary patterns in patients with Raynaud's phenomenon. An analysis of patterns discriminating patients with and without connective tissue disease. J Rheumatol 13:556-63,1986.

16. Caspary L, Schmees C, Schoetensack I, et al: Alterations of the nailfold capillary morphology associated with Raynaud phenomenon in patients with systemic lupus erythematosus. J Rheumatol 18: 559-66,1991.

17. Furtado RN, Pucinelli ML, Cristo VV, Andrade LE, Sato EI: Scleroderma-like nailfold capillaroscopic abnormalities are associated with anti-U1-RNP antibodies and Raynaud's phenomenon in SLE patients. Lupus 11:35-41,2002.

18. Spencer-Green G: Outcomes in primary Raynaud phenomenon: a meta-analysis of the frequency, rates, and predictors of transition to secondary diseases. Arch Intern Med 158:595-600,1998.

19. Luggen M, Belhorn L, Evans T, Fitzgerald O, Spencer-Green G: The evolution of Raynaud,s phenomenon: a longterm prospective study. J Rheumatol 22:2226-32,1995.

20. Priollet P, Vayssairat M, Housset E: How to classify Raynaud,s phenomenon. Am J Med 83:494-8,1987.

21. Masi AT, Rodnan GP, Medsger Jr TA, et al: Preliminary criteria for the classification of systemic sclerosis (scleroderma). Arthritis Rheum 23:581-90,1980. 
22. LeRoy EC, Medsger Jr. TA: Criteria for the classification of Early Systemic Sclerosis. J Rheumatol 28:1573-6,2001.

23. Lonzetti LS, Joyal F, Raynauld JP, et al: Updating the American College of Rheumatology preliminary classification criteria for systemic sclerosis: addition of severe nailfold capillaroscopy abnormalities markedly increases the sensitivity for limited scleroderma. Arthritis Rheum 44:735-6,2001.

24. Maricq HR, Spencer-Green G, LeRoy EC: Skin capillary abnormalities as indicators of organ involvement in scleroderma (systemic sclerosis), Raynaud's syndrome and dermatomyositis. Am J Med 61:862-70,1976.

25. Pucinelli MLC, Atra E, Sato EI, Andrade LEC: Nailfold cappilaroscopy in systemic sclerosis: correlations with involvement of lung and esophagus. Rev Bras Reumatol 35:136-42,1995.
26. Bredemeier M: A capilaroscopia periungueal pode sugerir atividade de doença pulmonar na esclerose sistêmica. Dissertação de Mestrado, Universidade Federal do Rio Grande do Sul, 2002.

27. Cutolo M, Sulli A, Pizzorni C, Accardo S: Nailfold videocapillaroscopy assessment of microvascular damage in systemic sclerosis. J Rheumatol 27:155-60,2000.

28. Cutolo M, Grassi W, Matucci Cerinic M: Raynaud's phenomenon and the role of capillaroscopy. Arthritis Rheum 48:3023-30, 2003.

29. Riccieri V, Rinaldi T, Spadaro A, et al: Interleukin-13 in systemic sclerosis: relationship to nailfold capillaroscopy abnormalities. Clin Rheumatol 22:102-6,2003. 